\title{
A REVIEW ON BIOCHEMICAL MECHANISM OF FATTY ACIDS SYNTHESIS AND OIL DEPOSITION IN BRASSICA AND ARABIDOPSIS
}

\author{
Mukhlesur Rahman \\ Department of Plant Sciences, North Dakota State University, Fargo, ND 58108-6050, USA
}

Received 2014-12-11; Revised 2014-12-22; Accepted 2014-12-28

\begin{abstract}
Oilseeds crops provide edible oil for humans' diet or oil for industrial purpose. Triacylglycerols (TAG) are the main components in seed oil which is formed by the esterification of fatty acids to glycerol back bone. In plants, fatty acid biosynthesis process initiated in plastid and ended in the endoplasmic reticulum. A series of biochemical steps are involved in TAG formation and many of the genes involved in this process have been identified. The quality of seed oil depends on the type of fatty acids esterified to glycerol. Finally, the TAGs are stored as lipid in seeds. The biosynthesis process of fatty acids and oil formation in seeds is reviewed in this manuscript.
\end{abstract}

Keywords: Fatty Acids, Biosynthesis, Triacylglycerols, Oil Formation

\section{INTRODUCTION}

Rapeseed/canola (Brassica napus L.) is the world's third most important edible oil crop, has become a major oilseed crop in Canada, Europe, Australia, China, USA and the Indian subcontinent. The quality of canola oil is determined primarily by its constituent fatty acids. The major fatty-acid constituents of Brassica oil are Palmitic acid (C16:0), Stearic acid (C18:0), Oleic (C18:1), Linoleic acid (C18:2), Linolenic acid (C18:3), Arachidic acid (C20:0), Eicosenoic acid (C20:1), Erucic acid (C22:1). In human diets, high levels of saturated fats (such as palmitic and stearic acid) have been positively correlated with cardiovascular disease. The levels of saturated fat in canola oil are usually less than $7 \%$ permitting canola to be labeled as low saturated fatty acid oil.

High-oleic-acid content in the seed oil of Brassica increases the thermostability of the oil, making it more suitable as cooking oil and therefore a desirable target for quality improvement. Oleic acid plays an important role for the biosynthesis of fatty acids. After an initial first desaturation it produces linoleic acid and after a second desaturation, it produces linolenic acid. Oleic acid can also undergo chain elongation to form ecosenoic acid and, subsequently, erucic acid (Sharma et al., 2002). In 1974, the first "Double Low" rapeseed variety "Tower" (which reduced both erucic acid and glucosinolates levels) was developed by the famous plant breeder Dr. Baldur Stefansson at the University of Manitoba, Canada. The development of a low erucic acid variety was made possible by the discovers of a low erucic acid variety "Liho", which had gene mutation for erucic acid biosynthesis in B. rapa (Stefansson and Hougen, 1964). In this case the mutant genes genetically blocked the biosynthetic pathway for the fatty acid elongation process and thus reduced the amount of erucic acid in seed. Erucic acid is one of the main fatty acids in rapeseed oil. Low erucic acid in rapeseed improves the quality of the oil, because high erucic acid is relatively low in digestibility and has been associated with health problems (Beare et al., 1963). On the other hand, High Erucic Acid Rapeseed (HEAR) has several potential applications in the oleo-chemical industry for the production of high temperature lubricants, nylon, plastics, slip and coating agents, soaps, painting inks, surfactants (Topfer et al., 1995).

Linolenic acid is a trienoic acid, a poly unsaturated fatty acid, easily oxidized, causing rancidity and offflavors and thus shortening the shelf life of the oil. Plant breeders have developed low linolenic acid canola cultivars that have increased the frying stability of the oil. Linolenic acid is synthesized either from the desaturation of linoleic acid (C18:2) and also perhaps by 
the elongation of roughanic acid (C16:3) (Tanhuanpaa and Schulman, 2002).

Triacylglycerols (TAG) are the main components in seed oil. To increase quantities of seed oil in oilseed crops it is important to understand the mechanism of TAG biosynthesis. Two different steps are involved for the synthesis of TAG as storage lipids in developing seeds. The first step is the production of acyl chains in the plastids and the second step is sequential incorporation of these acyl chains into glycerolipids by acyltransferases which forms TAG in the endoplasmic reticulum (Ohlrogge and Browse, 1995). TAG formation involves a series for biochemical steps with many genes are involved in controlling the reactions. Currently, most of the biochemical pathways for TAG synthesis have been reported and many of the genes involved in this process have been identified (Beisson et al., 2003). There is an evidence of using microarray technique in Arabidopsis seed development, that many genes are involved in the conversion of sugar to oil and these genes work in a coordinated fashion (Ruuska et al., 2002). It thus appears that many different regulatory factors are involved in controlling the expression of many of the genes in the oils biosynthesis pathway. In this review, I will discuss the different mechanism involved in fatty acid biosynthesis and oil production in plants especially in Brassica and Arabidopsis.

\subsection{Fatty Acid Biosynthesis}

Plastids are the major plant cell organelle for the initiation of the fatty acid biosynthesis process with this process completed in the endoplasmic reticulum (Harwood, 1988). The plastidial and all other cellular membranes in all cells are synthesized from these fatty acids. Some other plant parts primarily seeds, also produce TAG as storage oils to provide a source of energy for the developing seed after germination. Most of the fatty acids in plants have a chain length of 16 or 18 carbons. These fatty acids contain one to three double bonds all in a cis configuration. The major fatty acids in glycerolipids are palmitic acid (C16:0), oleic (C18:1), linoleic acid (C18:2), linolenic acid (C18:3), roughanic acid (C16:3) which comprise over $90 \%$ of the total fatty acids found in all plant membranes (Ohlrogge and Browse 1995).

There are rarely any free fatty acids found in any plant cells. The carboxyl group of free fatty acids is esterified to glycerol or otherwise modified to produce lipids and called glycerolipids. The glycerol has three different positions for the attachment of fatty acids and these positions are sn- 1, sn-2 and sn-3 (Fig. 1). The nature of glycerolipids depends upon the attachment of different fatty acids in these three different positions.
Triacylglycerol (TAG) is formed after all three positions on glycerol are esterified with fatty acids and stored as lipid in seeds (Fig. 1). Triacylglycerols are nonpolar nature and therefore referred to as neutral lipids.

Fatty acid biosynthesis in plant is a cyclic process consisting of a condensation reaction (1), a reduction reaction (2), a dehydration reaction (3) and finally, another reduction reaction (4) (Fig. 2). These reactions are catalyzed by $\beta$-ketoacyl-ACP synthase, by $\beta$ hydroxyacyl-ACP reductase, by $\beta$-hydroxy-ACP dehydratase and by enoyl-ACP reductase, respectively. Palmitoyl-ACP is the final product after 7 cycles of these reactions, which is then further elongated to stearoylACP by ß-ketoacyl-ACP synthase II (5) in conjunction with enzymes (2)-(4). The unsaturated oleoyl-ACP is produced after desaturation of stearoyl-ACP by the enzyme $\Delta^{9}$ desaturase (Fig. 2).

ATP and acetyl-CoA, NADPH and NADH are required for every two carbon addition to a growing acyl chain in the reactions catalysed by acetyl-CoA carboxylase and fatty acid synthetase (Slabas and Fawcett, 1992). In photosynthetic tissue, photosynthesis in chloroplasts provides the NADPH and ATP, required for fatty acid biosynthesis. In non-photosynthetic tissue, NADPH and ATP are imported by plastids or generated intraplastidially through carbohydrate oxidation or metabolite shuttles (Rawsthorne, 2002).

\section{BIOCHEMICAL ACTIVITIES IN PLASTID}

\subsection{Acetyl-CoA Synthesis}

Plastids contain a pool of acetyl-CoA, the source of carbon for the synthesis of fatty acids. The acetyl-CoA pool remains nearly constant in the size over the diurnal cycle, even though fatty acid biosynthesis is photosynthesis dependent, i.e., high during the light period and low in the dark period.

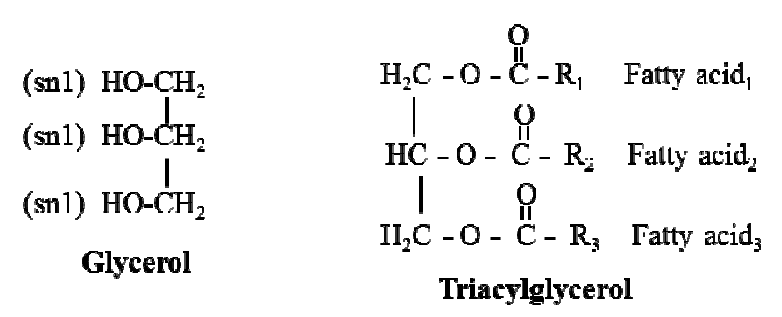

Fig. 1. Molecular structures of glycerol and triacylglycerol (adapted from http://www.scientificpsychic.com/fitness/fattyacids1.html access on February 20, 2014) 


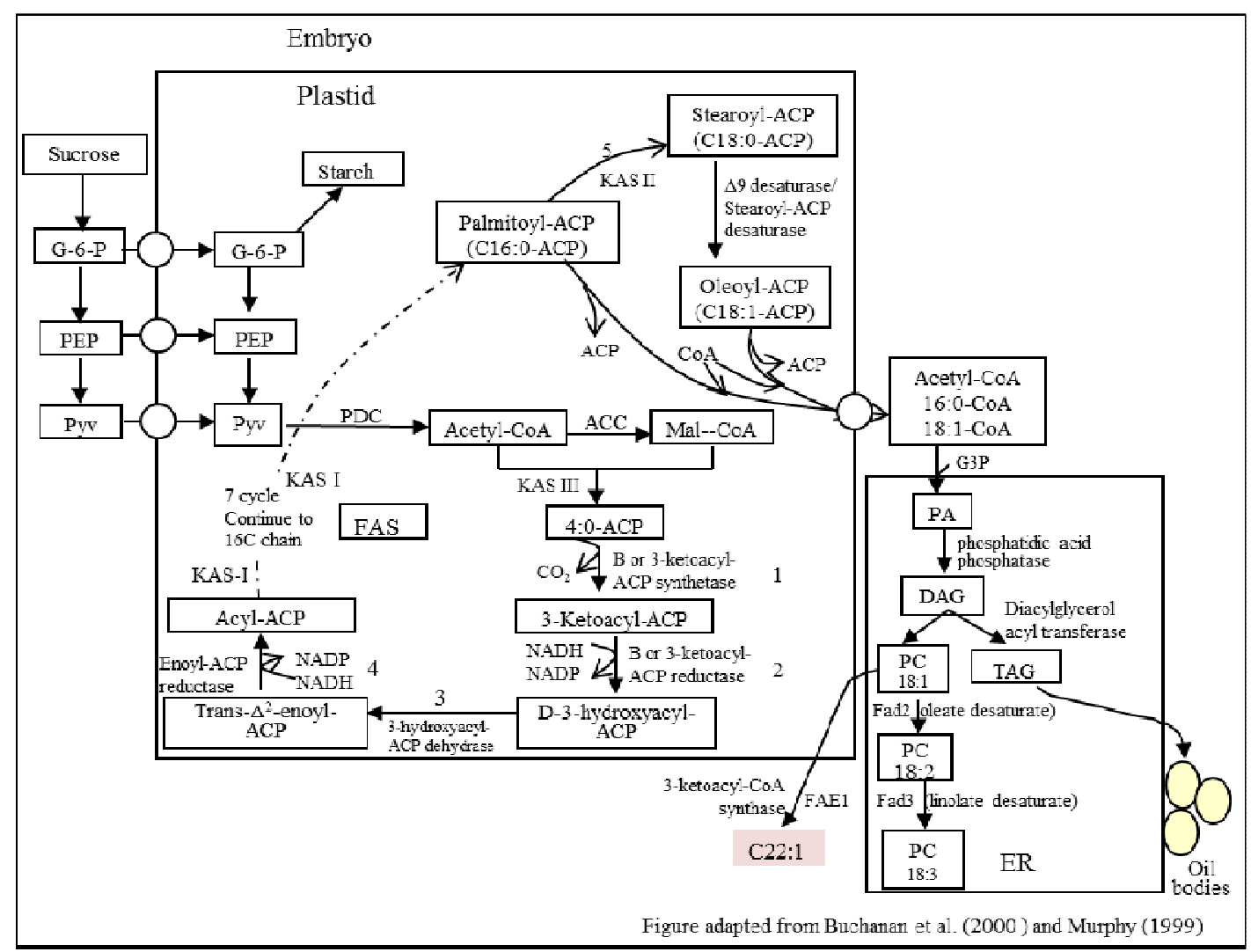

Fig. 2. Metabolic pathways of fatty acid and lipid biosynthesis in seed. In embryo, G-6-P, PEP and pyruvate may produce through glycolytic pathway in cytosol using sucrose. The plastid envelopes contain transporters that pass those products into plastid from cytosol. In oilseed, glycolytic pathway is also available and produces pyruvate. After enzymatic reaction of pyruvate, acetyl CoA is generated as the source of two carbon units for the synthesis of fatty acids. This synthesis requires a combined action of the enzyme complexes, Acetyl-CoA Carboxylase (ACC) and Fatty-Acid Synthase (FAS). Plant fatty acid synthetase is a cyclic process of a condensation reaction (1), reduction reaction (2), dehydration reaction (3) and finally another reduction reaction (4). Seven times repetition of this process produces $\mathrm{C}_{16}$ fatty acid. The first turn condensation reaction is catalysed by ketoacyl-ACP synthetase III (KAS III) and the next six turns of the cycle, the condensation reaction is catalysed by isoform of KAS I. Finally KAS II is used for the conversion of 16:0 to 18:0. Fatty acids release the ACP and attached to acyl-CoA derivatives and exported to the cytosol. In Endoplasmic Reticulum (ER), the acyl chains are incorporated into glycerolipids through acylation of Glycerol-3-Phosphate (G-3-P) by the action of the acyltransferases and produce Phosphatidic Acid (PA). Diacylglycerol (DAG) forms from the PA by enzymatic reaction with phosphatidic acid phosphatase. Phosphatidyl Choline (PC) and storage Triacylglycerol (TAG) are synthesized from PA. The enzymes involves for the desaturation of oleic acid to linoleic acid is $\Delta^{12}$ oleate desaturase (FAD2) and then linoleic acid to linolenic acid is $\omega$-3-Fatty Acid Desaturase (FAD3). Erucic acid synthesized from a elongation reaction from PC after condensation reaction catalyzed by 3-Ketoacyl-CoA Synthase (KAS). Mal-CoA: Malonyl-CoA; PEP: Phosphoenolpyruvate; PDC: Pyruvate Dehydrogenase Complex; ACP: Acyl Career Protein; PA: Phosphatidic Acid; DAG: Diacylglycerol; TAG: Triacylglycerol; PC: Phosphatidyl Choline; FAD: Fatty Acid Desaturase; FAE: Fatty Acid Elongase

Acetyl-CoA is thought to be generated from pyruvate after enzymatic reaction with plastidial Pyruvate Dehydrogenase (PDH) and PDH activity is usually high in non-green plastids (Ohlrogge and Browse, 1995). This mechanism for the generation of acetyl-CoA from pyruvate has been questioned. Since the low PDH activity found in chloroplasts is too little to explain the high rates of fatty acids biosynthesis observed (Ohlrogge and Browse, 1995). In contrast, the enzyme acetyl-CoA synthetase was found at very high levels in chloroplasts and, in a similar way, the amount of free acetate in chloroplasts was higher than that of pyruvate and other 
substrates for fatty acid biosynthesis in the chloroplasts (Ohlrogge and Browse, 1995). Free acetate is available in the cytosol and it enters into plastids and then, acetylCoA is generated from this acetate by acetyl-CoA synthetase. This enzyme has 5 to 15 times higher activity than that of in vivo fatty acid synthesis. It has also been proposed that the plastid acetyl-CoA pool in oilseeds is generated from cytoplasmic malate and glucosephosphate (Roughan and Ohlrogge, 1994).

A four possible pathways for the biosynthesis of acetyl-CoA in plastids was proposed (Rawsthorne, 2002). (1) Acetyl-CoA synthesis from free acetate after enzymatic reaction with Acetyl-CoA Synthetase (ACS). This is an ATP-dependent reaction; several authors measured the ACS activities in the chloroplast and reported that this enzymes property were consistent with its role in the biosynthesis of fatty acids (Rawsthorne, 2002). (2) From pyruvate to acetyl-CoA via the Pyruvate Dehydrogenase Complex (PDC). Pyruvate is imported from the cytosol or may be generated in the plastid by a glycolytic process. Two types of PDC have been reported in plants namely mitochondrial PDC and plastidial PDC. Several researchers have reported in that many species, the primary PDC activity occurs in plastids (Rawsthorne, 2002). (3) Acetyl-carnitine provides the acetate to form acetyl-CoA via the plastidial carnitine acetyltransferase reaction. This is a within the plastid reaction where acetate is transferred from acetyl-carnitine to acetyl-CoA. However, this proposal for acetyl-CoA biosynthesis has raised many questions and is therefore controversial (Rawsthorne, 2002). (4) Synthesis of acetyl-CoA via the ATP-Citrate Lyase (ACL) reaction. ACL activity was found predominantly in the leaves of rape (B. napus) and spinach, whereas this activity was found predominantly in the cytosol in tobacco and pea (Rangasamy and Ratledge, 2000). Therefore, this proposed pathway for the biosynthesis of acetyl-CoA is also controversial.

\subsection{Acetyl-CoA Carboxylase}

Conversion of acetyl-CoA to malonyl-CoA is catalyzed by Acetyl-CoA Carboxylase (ACC), the first committed step in the de novo fatty acid synthesis pathway. This is an ATP-dependent reaction. Plant acetyl-CoA carboxylase is a biotin-containing enzyme that malonyl-CoA produce in a two step reaction (Murphy, 1963) as follows:

$$
\begin{aligned}
& \mathrm{ATP}+\mathrm{CO} 2+\mathrm{BCC} \\
& \stackrel{\text { Biotin carboxylase }}{\longrightarrow} \mathrm{CO} 2-\mathrm{BCCP}+\mathrm{ADP}+\mathrm{Pi} \\
& \mathrm{CO} 2-\mathrm{BCCP}+\text { acetyl-CoA } \\
& \stackrel{\text { Carboxyltransferase }}{\longrightarrow} \mathrm{BCCP}+\text { Malonyl }-\mathrm{CoA}
\end{aligned}
$$

Knowledge on the type of ACC involvement in the production of malonyl-CoA has been gathering and developing. For example it has been identified that ACC has two forms in plants: Type I ACC, found in yeast and mammals is a large, multifunctional enzyme, while type II enzyme found in prokaryotes, is a multisubunit complex. The concept of plastidial ACC activity in higher plant has been clarified recently and it is now known that type II form of enzyme is responsible for ACC synthesis in most plants, but exceptionally in the Gramineae, this activity is due to a type I activity (Konishi et al., 1996).

The type I enzyme is assumed to be available in the cytoplasm of all higher plants (Egli et al., 1993). But this enzyme has also been found in the plastids of oilseed rape, although the activity of this enzyme in developing seed in less than $10 \%$ of the cellular total ACC type I enzyme (Schulte et al., 1997; Roesler et al., 1997). The activity of type I ACC enzymes in higher plants is thus complicated.

Biosynthesis of fatty acids from malonyl-CoA through ACC activity in leaves is regulated by the light reactions of photosynthesis. But whether the ACC in plant tissues produces storage oil or is required for oil production is contentious (Rawsthorne, 2002). ACC enzyme inhibitors in isolated barley and maize leaf chloroplasts and reported that ACC has a strong control over synthesis of fatty acid (Page et al., 1994). The amount of fatty acid in rapeseed increased up to $5 \%$ by increasing the total plastidial ACC activity by up to two times by targeting the type-I ACC to the plastid of developing seed embryos. Therefore, it has been concluded that ACC can regulate the amount of fatty acid biosynthesis in leaves.

\subsection{Acyl Carrier Protein}

Acyl Carrier Protein (ACP) arrives just before the initial process of storage lipid biosynthesis and plays a key role in fatty acid and lipid metabolism. It is a small, $9 \mathrm{kD}$ multifunctional, acidic protein. Acyl-ACP forms from a phosphopantetheine prosthetic group of ACP through a thioester linkage and takes part in the transportation, reduction, dehydration and elongation reactions of fatty acids. During the entire biosynthesis of fatty acids the growing fatty acid chain is bound to ACP and only released from the protein when it leaves the plastid (Fig. 2). After transfer to ACP, the malonylthioester enters into a series of condensation reactions with acyl-ACP (or acetyl-CoA) acceptors. These reactions result in the formation of a carbon-carbon bond and in the release of the $\mathrm{CO}_{2}$ that was added by the ACC reaction. In developing seeds of oilseed rape and soybean, it has been found that the rate of fatty acid synthesis is correlates with the levels of ACP concentration (Murphy, 1993). 


\subsection{Fatty Acid Synthetase}

The most important enzyme for fatty acid biosynthesis is Fatty Acid Synthetase (FAS). The component proteins of this enzyme can be isolated so as to retain active enzyme properties (Murphy, 1993). For the production of fatty acids three different condensing enzymes [also known as $\beta$ or 3-Ketoacyl-ACP Synthases (KAS)] are required. The first condensing enzyme is KAS III, which produces four carbon products from the condensation of acetyl-CoA and malonyl-ACP. The second condensing enzyme is KAS I, which is thought to increase the chain length from a four carbon chain to a 16 carbon chain (palmitoyl-ACP). Finally, the third condensing enzyme is KAS II, which elongates the 16 carbon palmitoyl-ACP product to 18 carbon stearoyl-ACP (Ohlrogge and Browse, 1995).

3-ketoacyl-ACP is the primary product of the condensation reaction of acetyl-CoA and malonyl-CoA. The enzyme 3-ketoacyl-ACP reductase reduces the 3ketoacyl-ACP at the carbonyl group, (with NADPH used as the electron donor). Dehydration is the next reaction catalyzed by hydroxyacyl-ACP dehydratase. Acyl-ACP is the product of this dehydration step. The enzyme enoyl-ACP reductase utilizes acyl-ACP as a substrate and seems for seven cycles to produce palmitoyl-ACP. Palmitoyl-ACP is the end product of this fatty acid biosynthesis process. Palmitoyl-ACP can be elongated further to generate Stearoyl-ACP (Fig. 2).

Different plant species contains a considerable diversity in the chain length of their fatty acid composition in their oil lipid. For example, the relative proportions of $\mathrm{C}_{16}: \mathrm{C}_{18}$ acids can vary from crop to crop (i.e. 45:42 in palm, 4:82 in oilseed rape and 11:82 in soybean). Because the conversion of $\mathrm{C}_{16}$ to $\mathrm{C}_{18}$ is controlled by the enzyme KAS II, these results indicate that this enzyme might have an important role in regulating of these fatty acids (Murphy, 1993). Therefore, two areas of interest have been arisen for the condensing enzyme. The first area contains on the fact that KAS III catalyses the initial reaction of plant fatty acid synthesis to form four carbon products from acetylCoA and Malonyl-CoA and offers a potential site for metabolic control. The second area contains on the fact that the activity of KAS II may have a key role in regulating seed oil quality by controlling the proportion of $\mathrm{C}_{16}: \mathrm{C}_{18}$ products during fatty acid biosynthesis.

\subsection{Termination of Fatty Acid Synthesis in Plastid}

The acyl group is the key building block for the elongation and termination of fatty acid synthesis. The termination of fatty acid elongation occurs when the acyl group is removed from ACP. Two different mechanisms for this termination process have been identified. The first mechanism, acyl-ACP is hydrolyzed by the enzyme acyl-ACP thioesterase and free fatty acid is released from the acyl chain. This is the most common mechanism for fatty acid elongation in plants. In the second mechanism, transfer of the fatty acid from ACP to glycerol-3-phosphate or to monoacylglycerol-3phosphate modulated by acyltransferases occurs in the plastid. One or two acyltransferases are involved in this process of chain elongation. The first acyltransferase prefers C18:1-ACP as a substrate and is a soluble enzyme present in the plastid. The second acyltransferase preferentially selects $\mathrm{C} 16: 0-\mathrm{ACP}$ and is an enzyme present on the inner envelope membrane of the chloroplast. The chloroplast membrane plays a key role for the transportation of the fatty acids using simple diffusion across the envelope. It is assumed that acylCoA synthetase, present on the outer membrane of chloroplast envelope, assembles an acyl-CoA thioester. This acyl-CoA thioester is then transferred to the endoplasmic reticulum to form glycerolipids using acyltransferase reactions. Recently small abundant proteins called acyl-CoA binding proteins have been identified and it is assumed that these binding proteins are involved in the transportation of acyl-CoA to the endoplasmic reticulum. The mechanism involved in this transportation process is unknown (Ohlrogge and Browse, 1995).

\section{EXTRA PLASTIDIAL ACTIVITIES}

\subsection{Endoplasmic Reticulum}

The Endoplasmic Reticulum (ER) is a major plant cell organelle that plays a key role in lipid biosynthesis and lipid storage. It is the port of entry of lipid into the endomembrane system. The ER serves specialized functions for the deposition of lipid storage compounds. This deposition is possible because the ER contains a number of soluble and membrane-associated enzymes and molecular chaperones, which are necessary for this process. Plant ER contains the necessary enzymes and proteins that are involved in the oil body biosynthesis and lipid storage process (Galili et al., 1998).

\subsection{Fatty Acid Desaturases}

Desaturases introduce double bonds at specific positions in the fatty acid chain are present on the ER membranes. Unsaturated $\mathrm{C}_{18}$ fatty acids, such as oleate, linoleate and linolenate are the most abundant fatty acids in many of the major edible oilseed crop species (for example canola, soybean, sunflower etc.). Aerobic desaturation of fatty acids produces these unsaturated fatty acids. In B. napus, the rate of desaturation is higher 
in plants grown in lower temperatures (e.g., $5^{\circ} \mathrm{C}$ ) compared to plants are grown in higher temperatures (e.g., $30^{\circ} \mathrm{C}$ ) (Williams et al., 1992). It has been reported that the palmitoyl- and oleoyl-acyl carrier proteins are the immediate products of fatty acid synthatase and stearoyl-acyl carrier protein desaturase in plastids (Murphy 1999). Different mechanisms are involved in the transportation of plasitdial 16:0 and 18:1 acyl chains to the endoplasmic reticulum for the production of glycerolipids. In the first mechanism, oleic acid is liberated from acyl-ACP thioesterase in the plastid and then re-esterified to CoA on the plastid envelop. The oleoyl-CoA is then associated with acyl-CoA pool in cytosol. These acyl groups may further go for esterification or acyl-exchange reaction in the cytosol. The acyl-CoA thioesters form a soluble pool in the cytosol which is used in the biosynthesis of Diacylglycerol (DAG) followed by Phosphatidylcholine (PC) or Triacylglycerol (TAG) (Williams et al., 2000).

Higher plants contain Phosphatidylcholine (PC) in their cells. This is the major phospholipids found in the plant cells. It is found in most non-plastidic membranes, the endoplasmic reticulum, the mitochondria, the plasma membrane and tonoplast and the outer-envelope membrane of the chloroplast. It plays a key role in glycerolipid biosynthesis and in the modification of membrane structures in leaves and is also involved in the biosynthesis of TAG in seeds of oilseed plants (Williams et al., 2000). It is located mostly in the endoplasmic reticulum. It has been identified that this is the major site of fatty acid desaturation and a precursor of chloroplastic lipids in leaves and in most plants. As similar role for PC has been found in oilseeds where PC appears to control the amount of unsaturated fatty acids in seed-oil (Williams et al., 2000). DAG is the presumed precursor of PC. Precursor DAG contains mainly oleic acid esterified to the $s n-1$ and $s n-2$ positions or palmitic acid esterified to the $s n-1$ position of DAG. Linoleic (18:2) and linolenic (18:3) acids are produced from the in situ desaturation of oleic acid (Fig. 3). The enzymes involved in the desaturation of oleic acid to linoleic acid are $\Delta^{12}$ oleate desaturase (encoded by the FAD2 gene) and for the further desaturation of linoleic acid to linolenic acid, $\omega-3$ fatty acid desaturase (encoded by the FAD3 gene).

Structural components of leaf membranes are produced from unsaturated fatty acids which are found in PC. These unsaturated fatty acids may also be used as a precursor for DAG used for the production of cytoplasmic phospholipids (e.g., phosphatidylethanolamine) and chloroplast lipids [Monogalactosyl Diacylglycerol (MGDG), Digalactosyl Diacylglycerol (DGDG) and Sulphoquinovosyl Diacylglycerol (SQDG)] (Fig. 3). A similar mechanism with some modifications has proposed to occur in seeds, where it has been found that some exchange of fatty acid between PC and the acyl-CoA occur before the synthesis of DAG and TAG (Triki et al., 1999). The amount of seed TAG and chloroplastic lipid depends on the amount of unsaturated fatty acid in DAG which is controlled by the desaturation of fatty acids in PC. The role of PC in desaturation and fatty acid exchange using in vivo ${ }^{14} \mathrm{C}$-labelling in leaves of Brassica napus was examine (Williams et al., 2000). The authors suggested that in the chloroplast newly formed saturated (palmitic acid, 16:0) and monounsaturated (oleic acid, 18:1) fatty acids are transferred into the endoplasmic reticulum for desaturation and incorporation into MGDG. Polyunsaturated fatty acids are generated in PC resulting from subsequent desaturation of the newly formed fatty acids. The acyl-CoA pool receives the unsaturated fatty acids to generate Diacylglycerol (DAG). It is assumed that these DAG containing unsaturated fatty acids are the precursors for the biosynthesis of other cellular glycerolipids (Williams et al., 2000).

Eight different desaturase mutant genes in Arabidopsis has been identified and characterized which provide a detailed insight of the desaturation gene in other crops (e.g., Brassica sp). These Arabidopsis mutants were identified from mutagenized populations and have been characterized by analyzing different lipid samples from leaves or seeds of mutagenized plants. The biochemical activities of these mutant genes have shown in the pathway in Fig. 3. The mutant genes responsible for specific fatty acid desaturases are designated $f a b 1$, fab2 and fad2 through fad8. Arabidopsis endoplasmic reticulum contains only two desaturase genes, oleate desaturase (FAD2) and linoleate desaturase (FAD3). The remaining eight desaturase genes are located in the plastids. In plastids, the gene FAD6 encodes oleate desaturase while the genes FAD7 and FAD8 encode linoleate desaturases. From the results of mutant analysis, it has found that FAD4 is responsible for the production of phosphatidylglycerol and FAD5 is involved in the formation of MGDG (Somerville and Browse, 1991). FAD2 and FAD3 genes present in the ER play an important role in the desaturation of fatty acids to control oil quality in the seed. The levels of oleic, linoleic and linolenic acid in seed oil are controlled by the enzymes encoded by FAD2 and FAD3 genes.

\subsection{Fatty Acid Elongation}

Erucic acid synthesis: Cruciferae crops, especially Brassica and Crambe species are major sources of erucic acid (cis-13-docosenoic acid). The pathway for erucic acid biosynthesis and the major reactions involved in this pathway has been well characterized. Oleic acid is the main precursor for the erucic acid biosynthesis via an elongation process in the developing embryos of Brassica napus, Crambe abyssinica, Simmondsia 
chinensis, Tropaeolum majus and Limnanthes alba (Bao et al., 1998). Erucic acid biosynthesis started initially in the plastid through the biosynthesis of oleate (18:1), which was then exported to cytosol and elongated via malonyl-CoA requiring elongases to form C-20 and subsequently C22 long chain monounsaturated fatty acids. These reactions usually occur in the endomembrane system. Many researchers reported that this elongation process occurs outside of the plastid which is related to the oil bodies or microsomal membrane (Bao et al., 1998). The general accepted hypothesis for the erucic acid biosynthesis is as follows: in the plastid oleoyl-ACP is the end product of fatty acids biosynthesis process, which releases the Acyl Carrier Protein (ACP) and binds with CoA to form oleoyl-CoA which then moves to the cytosol. Oleoyl-CoA is the precursor for the elongation process to form erucic acid and this process occurs in endoplasmic reticulum. Malonyl-CoA is the source of carbon for this elongation process (Bao et al., 1998).

Elongation process: In higher plants, it has been demonstrated that at least two types of elongases are available for the biosynthesis of erucic acid; these are the ATP-dependent elongase and the acyl-CoA elongase. An unrecognized endogenous compound is elongated through ATP-dependent elongation process. ATP is not required in the acyl-CoA elongation process, which uses acyl-CoA for the biosynthesis of erucic acid (Juliette et al., 2001). It was observed in rapeseed microsomes that high rates of oleoyl-CoA elongase activity reduced the rate of ATP-dependent elongation; on the other hand, high rates of ATP-dependent elongase activity limited the rate of oleoyl-CoA elongation (Juliette et al., 2001). These results indicated that oleoyl-CoA elongation and the ATP-dependent elongation processes do not act in the same plant tissue at the same time.

The elongation process has four different steps. The first step is the condensation of oleoyl-CoA to malonylCoA to form a 3-ketoacyl-CoA, the second step is the reduction of the 3-ketoacyl-CoA to produce3hydroxyacyl-CoA, the third step is the dehydration of the 3 -hydroxyacyl-CoA to form trans-(2,3)-enoyl-CoA and the fourth and final step is further reduction of the trans(2,3)-enoyl-CoA (Juliette et al., 2005). These reactions are catalyzed by four different enzymes 3-ketoacyl-CoA synthase, 3-ketoacyl-CoA reductase, 3-hydroxyacyl-CoA dehydratase and trans-2,3-enoyl-CoA reductase, respectively (Fig. 4).

The 3-Ketoacyl-CoA Synthase enzyme (KCS) is the major catalyzing enzyme in the condensation reaction for the chain length of fatty acid to produce Very Long Chain Fatty Acid (VLCFA) in seed oils (Juliette et al., 2005). The reactions involved in this elongation process have been characterized biochemically, but gene expression and characterization of gene products still remains unknown. In Arabidopsis thaliana and jojoba, it has been reported that the gene FAE1 encodes 3ketoacyl-CoA synthase, the first committed enzyme for fatty acid elongation (Juliette et al., 2005).

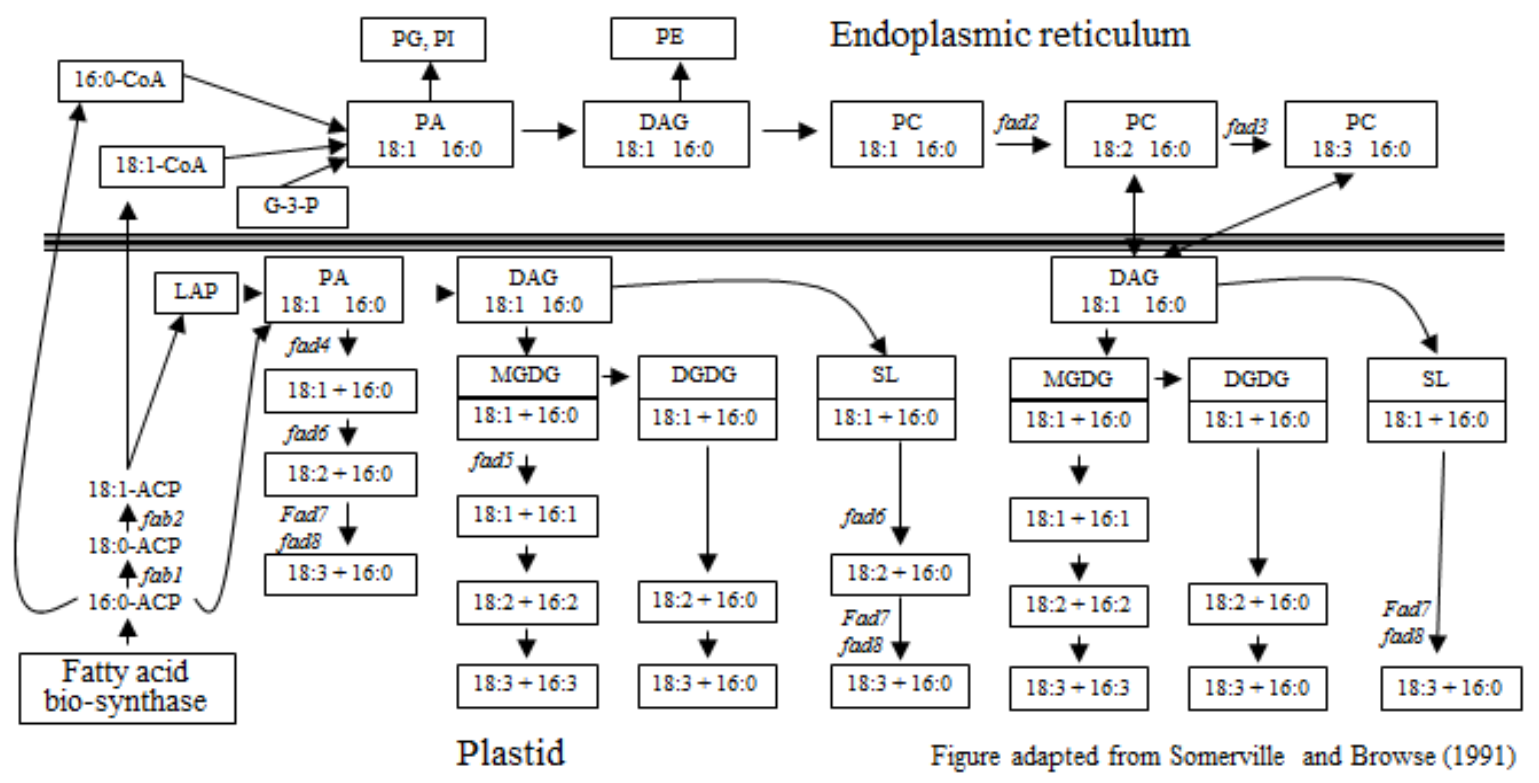

Fig. 3.Diagram of Fatty acid synthesis and glycerolipid assembly in Arabidopsis leaves. DAG: Diacylglycerol; DGDG: Digalactosyldiacylglycerol; G3P: Glucose-3-Phosphate; LPA: Lysophosphadic Acid; PA: Phosphatidic Acid; PC: Phosphatidylcholine; PE: Phosphatidyethanolamine; PG: Phosphatidylglycerol; PI: Phosphatidylinositol; MGDG: Monogalactosyldiacylglycerol; SQDG: Sulfoquinovosyldiacylglycerol 


\begin{tabular}{|c|c|c|c|c|c|c|}
\hline $\begin{array}{c}\text { C18:1-CoA } \\
+ \\
\text { Malonyl-CoA }\end{array}$ & synthase & $\begin{array}{l}\text { 3-ketoacyl } \\
\text {-CoA }\end{array}$ & reductase & $\begin{array}{l}\text { 3-hydroxy } \\
\text { acyl-CoA }\end{array}$ & $\underset{\text { dehydratase }}{\stackrel{\text { 3-hyroxyacyl-CoA }}{\longrightarrow}}$ & $\begin{array}{l}\text { Trans-2,3- } \\
\text { enoyl-CoA }\end{array}$ \\
\hline
\end{tabular}

Fig. 4. Fatty acid elongation process

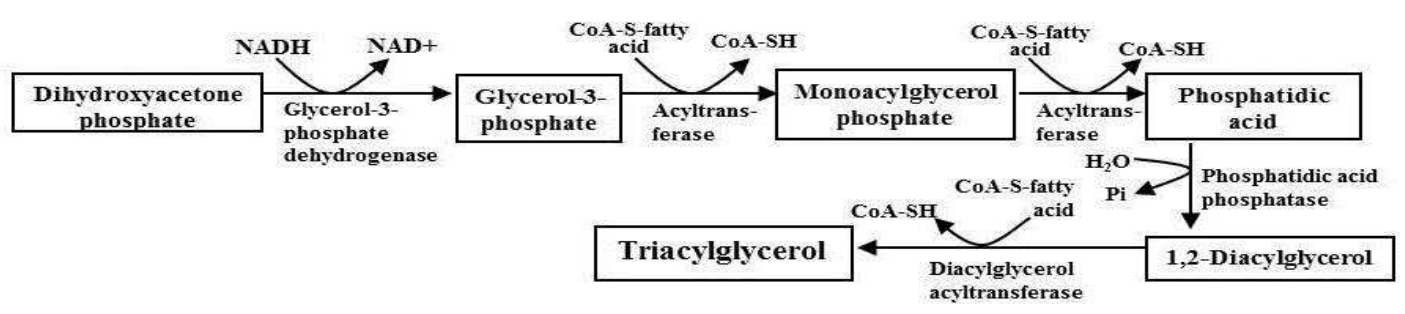

Fig. 5. Biochemical pathway of triglycerol synthesis (Kennedy pathway). [Figure adapted and modified from: Murphy (1993) and internet http://biocyc.org/META/new-image?type=PATHWAY\&object=TRIGLSYN-PWY]

In the embryoes of $B$. napus two homologous sequences of the FAE1 gene (Bn-FAE1.1 and Bn-FAE1.2) have been characterized. These two homologues showed a similarity of $98.2 \%$ amino acids sequence of the encoded proteins (Juliette et al., 2005). 3-ketoacyl-CoA synthase controlled the levels of very long chain fatty acids in seed oils of Arabidopsis (Millar and Kunst, 1997). Therefore, to increase the levels of erucic acid for high erucic acid rapeseed breeding it is necessary to know the role of FAE1 gene during the developmental process of embryo in seed. The gene encoding the enzyme 3ketoacyl-CoA reductase has been identified in $B$. napus and named as Bn-kcr (Bn-kcrl and Bn-kcr2). FAE1 and $\mathrm{Bn}-\mathrm{KCR}$ are expressed simultaneously in $B$. napus during seed development, suggesting that expression of both genes is directly or indirectly linked (Juliette et al., 2005).

\subsection{Triacylglycerol Synthesis}

Triacylglycerols (TAG) are formed by the Kennedy pathway (Fig. 5). The main pathway for synthesis of triacylglycerol is thought to involve in esterification of three fatty acids onto a glycerol backbone. Saturated fatty acids are mostly abundant in TAG. Biosynthesis of TAG depends on three consecutive reactions, formation of Phosphatidic Acid (PA) from glycerol-3-phosphate, followed by the formation of Diacylglycerol (DAG) from PA and finally TAG is formed from DAG (Fig. 5). For the synthesis of PA, fatty acids are sequentially transferred to the positions 1 and 2 of glycerol-3phosphate. DAG is formed from PA by the dephosphorylation of PA. In the final step, a third fatty acid is transferred to the vacant position 3 of DAG. Diacylglycerol acyltransferase is the most important enzyme, involved in the formation of TAG from DAG (Ohlrogge and Browse, 1995). Lipids synthesized within the ER always have 18-carbon fatty acid esterified at the sn-2 position of the glycerol backbone, whereas lipids synthesized in the chloroplast always have 16-carbon fatty acids esterified at the sn-2 position of the glycerol backbone (Somerville and Browse, 1996).

The esterification of fatty acids onto the glycerol backbone is not an easy process. In oilseed crops fatty acids generated in the plastids undergo further elongation or desaturation and then be esterified to produce TAG. Therefore, these fatty acids are not immediate precursors for the biosynthesis of TAG. The Acyl chains generated in plastid are converted into Phosphatidyl Choline (PC), the substrate for the desaturation or modification of fatty acids. Two mechanisms are involved to make the availability of these modified fatty acids for the formation of TAG. In the first mechanism, it is assumed that the acyl$\mathrm{CoA}$ is released from combined reverse and forward reactions of acyl-CoA: PC acyltransferase (Ohlrogge and Browse, 1995) which is then used for TAG biosynthesis. This reaction permits the newly synthesized oleic acid in the plastids to move into the PC for further desaturation or modification. The desaturated or modified fatty acid is then be used for the formation of TAG or other lipids. In the second mechanism, PC may release its whole DAG part for the biosynthesis of TAG (Ohlrogge and Browse, 1995).

In oilseed crops, the compositions of different fatty acids in seed storage oils are more variable than those are in membrane glycerolipids. In storage oils more than 300 different fatty acids have been observed in seed TAG [6]. The structures of these fatty acids. 


\section{OIL BODIES}

\subsection{Structure and Composition}

All living organisms utilize lipids and stored in their subcellular compartments for their active metabolism. In most plants, lipids are found in the seed as storage lipids in the form of TAGs (Galili et al., 1998). In the endoplasmic reticulum, TAG is synthesized by the enzymatic reaction with diacylglycerol acyltransferase. TAGs are mostly found in small distinct organelles which are called Oil Bodies (OBs) or oleosomes. Oleosins are small proteins of 0.5-2.0 $\mu \mathrm{m}$ diameter with a molecular weight of about 15 to $26 \mathrm{kD}$, present in seeds. The oleosins almost cover the surface of the OB. Amount of oleosins in seed could be very high with high levels of oils and small sized OBs. Arabidopsis thaliana seeds contain high levels of oil (about 40\%) and small sized OBs (about $0.5 \mu \mathrm{m}$ ) with $10 \%$ of the seed proteins found as oleosins. The size and shape of OB depends on the proportion of oil in the oleosins. The major components of OB are oleosins, TAGs and Phospholipids (PLs). TAGs are deposited inside the OB and surrounded by Phospholipids (PLs) and oleosins. The outer layer of the OB is composed of structural protein (oleosins) and phospholipids and therefore the structure of OB is stable.

\subsection{Biogenesis of Oil Bodies}

It has been postulated that oil bodies and their component TAGs, PLs and oleosins are synthesized on the Endoplasmic Reticulum (ER). The enzyme which catalyzes the reaction for TAG biosynthesis is diacylglycerol acyltransferase (DAGAT), located on the rough ER (Hsieh and Huang, 2004). A model for the biosynthesis of oil bodies on rough ER which included the synthesis of TAG, oleosins and PLs on the ER, followed by the assembling of those components in the cytoplasm in maturing seeds and then formed into oil bodies was proposed by (Galili et al., 1998). This model was agreed by (Herman, 1987) and (Tzen et al., 1993), since they reported that oleosins and storage oil are synthesized coordinately in maturing maize and soybean seeds. The in vitro translation and translocation of oleosins mRNAs showed that oil bodies were deposited to the microsomal membranes and at the same time oleosins were also integrated into microsomal membranes. There is no evidence of integration of oleosins into oil body monolayer membranes and oil bodies are synthesized in the sub-domain of the ER (Galili et al., 1998). This result stated that oleosins are synthesized and integrated into ER independently and then assembled in oil bodies.

The biosynthesis process of oleosins has been difficult to study because several genes are responsible for this process and these genes encode several protein products with different molecular weight. For example in rapeseed and soybean at least 6-8 copies of oleosins genes and in Arabidopsis, 4-5 oleosins genes have been identified and those genes encodes proteins with size from $19 \mathrm{kDa}$ to $24 \mathrm{kDa}$ (Sarmiento et al., 1997).

A model for lipid accumulation in B. napus was proposed by (Lacey and Hills, 1996), where TAGs are generated in the sub-domains of ER and deposited within phospholipids bilayer which is the site for oleosin insertion into ER. In the early stage of seed development the levels of TAGs is higher than those of oleosins and oleosins can not completely cover the oil body. Therefore, these oleosins merge with other free oleosin to make a full size of the oil body. This result indicated that oleosins are the major component for the full size of oil body (Galili et al., 1998).

\section{SUMMARY}

Because of the importance of plant seed oils as sources of edible oils and industrial oils, there is continuing interest in the use of genetic technology to produce novel fatty acids and related materials in plants. The major biochemical pathway of plant fatty acids biosynthesis and synthesis of glycerolipids are now mostly well understood. Most oil/lipid contains fatty acids esterified to glycerol. Fatty acids biosynthesis in plant takes place exclusively in the plastid. The synthesis of fatty acids begins with the carboxylation of acetylCoA to malonyl-CoA by acetyl-CoA carboxylase. This is the first committed step of fatty acids biosynthesis and is a likely site regulation of the whole pathway. AcetylCoA and malonyl-CoA are subsequently converted into fatty acids by series of reaction that add two carbons at a time to a growing chain. Repetitive condensation of 2carbon units for seven times yields C16:0 fatty acids. Most of the C16:0 fatty acids elongated to C18:0 (by using 3-ketoacyl-ACP synthetase III), but some is used for the biosynthesis of lipid within the chloroplast. Fatty acid biosynthesis can be terminated by several different reactions, including hydrolysis of the thioester bond, transfer of the acyl group to a glycerolipid and acyl desaturation. Acyl carrier protein transports the intermediates of fatty acid for the reduction, dehydration and elongation reactions. 
The acyl-CoAs are the primary substrates for fatty acids biosynthesis in the endoplasmic reticulum or in the plastid. The first steps of glycerolipid synthesis are two acylation reactions that transfer fatty acids to glycerol-3phosphate to form Phosphatidic Acid (PA). Diacylglycerol is produced from PA by a specific phosphatase. Phosphatidylcholine (PC) is formed from DAG which mainly contains oleic acid and palmitic acid. Desaturation of oleic acid in PC produces linoleic acid (enzyme encoded by gene FAD2) and linoleic acid to linolenic acids (enzyme encoded by gene FAD3). On the other hand 3-Ketoacyl-CoA Synthase (KCS) (encoded by gene FAE1) catalyze the condensation reaction, plays a key role for the elongation of oleic acid to erucic acid, occurs in Arabidopsis, as it does in rapeseed and other cruciferous oilseeds. Obviously, synthesis of DAG via PA does the final acylation of DAG to form Triacylglycerol (TAG) and the enzyme catalyzes for the synthesis of TAG is diacylglycerol acyltransferase. Finally, TAG, phospholipids and oleosins are synthesized on the ER and forms oil body, which is stored in seed.

\section{FUTURE PROSPECTS}

The amount of C18:1 and C22:1 in seeds is variable from species to species and even from variety to variety in a same species (e.g., high oleic and low oleic acid content canola varieties, high or low erucic acid content rapeseed varieties). These amount of oleic and erucic acids depend on how much free fatty acid transported from the plastid. Acyl-CoA binding protein involves in transportation of fatty acid to ER but the mechanism of this transportation is still unknown and need to know more for getting better insight of this biosynthesis process.

The membrane-bound acyl-CoA elongase complex is a key enzyme responsible for erucoyl-CoA synthesis. Four putative genes encoding the four moieties of this complex in B. napus seeds and two genes have been characterized, the Bn-fael gene, which encodes the 3ketoacyl-CoA synthase and Bn-kcr gene encodes 3ketoacyl-CoA reductase in $B$. napus seeds. Coexpression of Bn-fael and Bn-kcr is observed in B. napus during seed development. The genes encoding the other two enzymes (3-hydroxyacyl-CoA dehydratase and trans-2,3-enoyl-CoA reductase) have not been identified yet which could be a good study to find out the detail pathway for erucic acid synthesis.

The role of the plant ER has been studied extensively, but the role of ER in the biogenesis of oil bodies is not clear and particularly in the maturation of oleosins protein in seed. The way of integration of oleosins into the membrane of the ER and the way of the formation of oil body from this membrane is still questioned. In ER, multigene families may encode certain molecule and whether the integration of those molecules into the ER membrane is similar to those of integration of oleosins into ER membranes is unknown. Further studies are needed to determine the specific role of the individual members of these families in the biogenesis and functions of the ER.

\section{CONCLUSION}

Fatty acids biosysthesis pathway in Arabidopsis and Brassica has been discuss in this review article. Triacylglycerols (TAG) are formed by the Kennedy pathway through esterification of fatty acids to glycerol back bone and stored as lipid in seeds. Therefore, the quality of vegetable oil depends on the type of fatty acids esterified onto glycerol. This review will help the oilseed crop breeder and geneticists to understand the oil formation mechanism in seeds and to develop breeding strategy for the improvement of oilseed crops.

\section{ACKNOWLEDGEMENT}

The author gratefully acknowledge the financial support for the this project from National Institute of Food and Agriculture (under North Central Region Canola Research Grants).

\subsection{Ethics}

This article is original and contains unpublished material. The corresponding author confirms that all of the other authors have read and approved the manuscript and no ethical issues involved.

\section{REFERENCES}

Bao, X., M. Pollard and J. Ohlrogge, 1998. The biosynthesis of erucic acid in developing embryos of Brassica rapa. Plant Physiol., 118: 183-190. DOI: 10.1104/pp.118.1.183

Beare, J.L., J.A. Campbell, C.G. Youngs and B.M. Craig, 1963. Effects of saturated fat in rats fed rapeseed oil. Can. J. Biochem. Physiol., 41: 605-612.

Beisson, F., A.J.K. Koo, S. Ruuska, J. Schwender and M. Pollard et al., 2003. Arabidopsis genes involved in acyl lipid metabolism. A 2003 Census of the Candidates, a Study of the distribution of expressed sequence tags in organs and a web-based database. Plant Physiol., 132: 681-697.

DOI: $10.1104 /$ pp.103.022988 
Egli, M.A., B.G. Geng22enbach, J.W. Gronwald, D.A. Somers and D.L. Wyse, 1993. Characterization of maize acety 1-coenzyme a car boxy lase. Plant Physiol., 101: 499-506.

Galili, G., S.G. Champa and C. Aldo, 1998. The endoplasmic reticulum of plant cells and its role in protein maturation and biogenesis of oil bodies. Plant Molecular Biology, 38: 1-29. DOI: $10.1023 / \mathrm{A}: 1006011919671$

Harwood, J.L., 1988. Fatty acid metabolism. Annu. Rev. Plant Physiol. Plant Molecular Biology, 39: 101-138.

DOI: 10.1146/annurev.pp.39.060188.000533

Herman, E.M., 1987. Immunogold-localization and synthesis of an oil-body membrane protein in developing soybean seeds. Planta, 172: 336-345. DOI: $10.1007 / \mathrm{BF} 00398662$

Hsieh, K. and A.H.C. Huang, 2004. Endoplasmic reticulum, oleosins and oils in seeds and tapetum cells. Plant Physiol., 136: 3427-3434.

DOI: $10.1104 / p p .104 .051060$

Juliette, P., D. Wilfrid, C. Patricia, C. Sylvette and B. Annick et al., 2005. Temporal gene expression of 3ketoacyl-CoA reductase is different in high and in low erucic acid Brassica napus cultivars during seed development. Biochimica et Biophysica Acta, 1687: 152-163. DOI: 10.1016/j.bbalip.2004.11.014

Juliette, P., G. Bertrand, C. Patricia, D. Wilfrid and R. Thomas et al., 2001. Acyl-CoA elongase expression during seed development in Brassica napus. Biochimica et Biophysica Acta, 1533: 141-152. DOI: 10.1016/S1388-1981(01)00152-4

Konishi, T., K. Shinohara, K. Yamada and Y. Sasaki, 1996. Acetyl-CoA carboxylase in higher plants: Most plants other than gramineae have both the prokaryotic and the eukaryotic forms of this enzyme. Plant Cell Physiol., 37: 117-122.

DOI: 10.1093/oxfordjournals.pcp.a028920

Lacey, D.J. and M.J. Hills, 1996. Heterogeneity of the endoplasmic reticulum with respect to lipid synthesis in developing seeds of Brassica napus L. Planta, 199: 545-551. DOI: 10.1007/BF00195185

Millar, A.A. and L. Kunst, 1997. Very-long-chain fatty acid biosynthesis is controlled through the expression and specificity of the condensing enzyme. Plant J., 12: 121-131.

DOI: 10.1046/j.1365-313X.1997.12010121.x

Murphy, D.J., 1993. Designer Oil Crops, VCH Verlagsgesellschaft mbH, Weinheim, Germany and VCH Publishers Inc., New York, USA.
Murphy, D.J., 1999. Production of novel oils in plants. Current Opinion Biotect., 10, 175-180. DOI: 10.1016/S0958-1669(99)80031-7

Ohlrogge, J. and J. Browse, 1995. Lipid biosynthesis. Plant Cell, 7: 957-970. DOI: 10.1105/tpc.7.7.957

Page, R.A., S. Okada and J.L. Harwood, 1994. AcetylCoA carboxylase exerts strong flux control over lipid synthesis in plants. Biochimica et Biophysica Acta, 1210: 369-372.

DOI: 10.1016/0005-2760(94)90242-9

Rangasamy, D. and C. Ratledge, 2000. Compartmentation of ATP: Citrate lyase in plants. Plant Physiol., 122: 1225-1230. DOI: $10.1104 /$ pp.122.4.1225

Rawsthorne, S., 2002. Carbon flux and fatty acid synthesis in plants. Progress Lipid Res., 41: 182-196. DOI: 10.1016/S0163-7827(01)00023-6

Roesler, K., D. Shintani, L. Savage, S. Boddupalli and J.B. Ohlrogge, 1997. Targeting of the arabidopsis homomeric acetyl-coenzyme a carboxylase to plastids of rapeseeds. J. Plant Physiol., 113: 75-81. DOI: $10.1104 /$ pp.113.1.75

Roughan, P.G. and J.B. Ohlrogge, 1994. On the assay of Acetyl-CoA synthetase activity in chloroplasts and leaf extracts. Analytical Biochemis., 216: 77-82. DOI: 10.1006/abio.1994.1010

Ruuska, S.A., T. Girke, C. Benning and J.B. Ohlrogge, 2002. Contrapuntal networks of gene expression during Arabidopsis seed filling. Plant Cell, 14: 1191-1206. DOI: $10.1105 /$ tpc.000877

Sarmiento, C., J.H.E. Ross, E. Herman and D.J. Murphy, 1997. Expression and subcellular targeting of a soybean oleosin in transgenic rapeseed. Implications for the mechanism of oil-body formation in seeds. Plant J., 11: 783-796.

DOI: 10.1046/j.1365-313X.1997.11040783.x

Schulte, W., R. Topfer, R. Stracke, J. Schell and N. Martini, 1997. Multi-functional acetyl-CoA carboxylase from Brassica napus is encoded by a multi-gene family: Indication for plastidic localization of at least one isoform. Proc. Natl. Acad Sci. USA, 94: 3465-3470. DOI: $10.1073 /$ pnas.94.7.3465

Sharma, R., A.K. Aggarwal, R. Kumar, T. Mohapatra and R.P. Sharma, 2002. Construction of an RAPD linkage map and localization of QTLs for oleic acid level using recombinant inbreds in mustard (Brassica juncea). Genome, 45: 467-472.

DOI: $10.1139 / \mathrm{g} 02-001$ 
Slabas, A.R. and T. Fawcett, 1992. The biochemistry and molecular biology of plant lipid biosynthesis. Plant Mol. Biology, 19: 169-191. DOI: $10.1007 / \mathrm{BF} 00015613$

Somerville, C. and J. Browse, 1991. Plant lipids: Metabolism, mutants and membranes. Science, 252: 80-87. DOI: $10.1126 /$ science. 252.5002 .80

Somerville, C. and J. Browse, 1996. Dissecting desaturation: Plants prove advantageous. Trends Cell Biology, 6: 148-153.

DOI: 10.1016/0962-8924(96)10002-7

Stefansson, B.R. and F.W. Hougen, 1964. Selection of rape plants (Brassica napus) with seed oil practically free from erucic acid. Can. J. Plant Sci., 44: 359-364 DOI: $10.4141 /$ cjps64-069

Tanhuanpaa, P. and A. Schulman, 2002. Mapping of genes affecting linolenic acid content in Brassica rapa ssp. Oleifera. Molecular Breeding, 10: 51-62. DOI: 10.1023/A:1020357211089

Topfer, R., M. Nartini and J. Schell, 1995. Modification of plant lipid synthesis. Science, 268: 681-686.

DOI: $10.1126 /$ science.268.5211.681
Triki, S., C. Demandre and P. Mazliak, 1999. Biosynthesis of triacylglycerols by developing sunflower seed microsomes. Phytochemistry, 52: 55-62. DOI: 10.1016/S0031-9422(99)00064-3

Tzen, J.T.C., Y.Z. Cao, C. Ratnayake and A.H.C. Huang, 1993. Lipids, proteins and structure of seed oil bodies from diverse species. Plant Physiol., 101: 267-276. DOI: 10.1104/pp.101.1.267

Williams, J.P., V. Imperial, M.U. Khan and J.N. Hodson, 2000. The role of phosphatidylcholine in fatty acid exchange and desaturation in Brassica napus $\mathrm{L}$. leaves. Biochem. J., 349: 127-133. DOI: 10.1042/0264-6021:3490127

Williams, J.P., M.U. Khan and D. Wong, 1992. Low temperature-induced fatty acid desaturation in Brassica napus: Thermal deactivation and reactivation of the process. Biochim. Biophys Acta, 1128: 275-279.

DOI: $10.1016 / 0005-2760(92) 90318-\mathrm{P}$ 